\title{
UNIVALENT LOGHARMONIC RING MAPPINGS
}

\author{
ZAYID ABDULHADI, WALTER HENGARTNER, AND JAN SZYNAL
}

(Communicated by Clifford J. Earle, Jr.)

\begin{abstract}
Univalent logharmonic ring mappings are characterized in terms of univalent starlike mappings. An existence and uniqueness theorem is also given.
\end{abstract}

\section{INTRODUCTION}

Let $D$ be a domain of $\mathrm{C}$ and denote by $H(D)$ the set of all analytic functions on $D$. A logharmonic mapping is a solution of the nonlinear elliptic partial differential equation

$$
\overline{f_{\bar{z}}}=(a \bar{f} / f) f_{z},
$$

where the second dilatation function $a$ is in $H(D)$ and $|a(z)|<1$ for all $z \in D$. If $f$ does not vanish in $D$, then $f$ is of the form

$$
f=H \bar{G},
$$

where $H$ and $G$ are locally analytic (possibly multivalued) functions on $D$. On the other hand, if $f$ vanishes at $z_{0}$ but is not identically zero, then $f$ admits the local representation

$$
f(z)=\left(z-z_{0}\right)^{m}\left|z-z_{0}\right|^{2 \beta} h(z) \overline{g(z)}
$$

where $m \in N, \operatorname{Re} \beta>-m / 2$, and $h$ and $g$ are analytic in a neighbourhood of $z_{0}$ (see, e.g., [1]).

Univalent logharmonic mappings defined on the unit disk $U$ have been studied in $[1,2]$.

In this paper we investigate the family $\mathscr{A}_{r}$ of all univalent logharmonic mappings which map an annulus $A(r, 1)=\{z: r<|z|<1\}, 0<r<1$, onto an annulus $A(R, 1)$ for some $R \in[0,1)$ and which satisfy the condition $\frac{1}{2 \pi} \int_{|z|=\rho} d \arg f\left(\rho e^{i t}\right)=1$ for all $\rho \in(r, 1)$. The last condition says that the outer boundary corresponds to the outer boundary. We call an element $f \in \mathscr{A}_{r}$ a univalent logharmonic ring mapping.

If $a \equiv 0$, then $R=r$ and $f(z)=e^{i \alpha} z, \alpha \in R$, are the only mappings in $\mathscr{A}_{r}$. In the case of univalent harmonic mappings from $A(r, 1)$ onto $A(R, 1)$ it is possible that $R=0$; for example, $\left[1 /\left(1-r^{2}\right)\right]\left[z-r^{2} / \bar{z}\right]$ has this property. However, Nitsche [11] has shown that there is an $R_{0}(r)<1$ such that there is

Received by the editors February 27, 1991.

1991 Mathematics Subject Classification. Primary 30C55. 
no univalent harmonic mapping from $A(r, 1)$ onto $A(R, 1)$ whenever $R_{0}<$ $R<1$.

There is no univalent logharmonic mappings from $A(r, 1), 0<r<1$, onto $A(0,1)$. This is a direct consequence of $(2.1)$ in our Theorem 2.1. But, on the other hand, we have for $R$ neither a positive lower bound nor a uniform upper bound strictly less than one. Indeed, $f(z)=z|z|^{2 \beta}, \operatorname{Re} \beta>-\frac{1}{2}$, is univalent on $A(r, 1)$ and its image is $A\left(r^{1+2 \operatorname{Re} \beta}, 1\right)$.

Unlike the case of univalent harmonic mappings, univalent logharmonic ring mappings need not have a continuous extension onto the closure of $A(r, 1)$. Indeed,

$$
f(z)=z(\overline{z-1}) /(1-z)
$$

is a univalent logharmonic ring mapping from $A\left(\frac{1}{2}, 1\right)$ onto itself whose cluster sets on the outer boundary are $C\left(f, e^{i t}\right)=\{-1\}$, if $z=e^{i t}, 0<t<2 \pi$, and $C(f, 1)=\{w ;|w|=1\}$.

Theorems 2.1 and 2.2 give a complete characterization of univalent logharmonic mappings in $\mathscr{A}_{r}$. It turns out that these are exactly the mappings of the form

$$
f(z)=\left(\frac{\varphi(z)}{|\varphi(z)|}|z|^{2 \gamma}\right),
$$

where $\operatorname{Re} \gamma>0, \varphi \in H(A(r, 1))$, and where $p(z)=z \varphi^{\prime}(z) / \varphi(z)=1+$ $\sum_{k \in Z \backslash\{0\}} p_{k} z^{k}$ satisfies $\operatorname{Re} p(z)>0$ on $A(r, 1)$. It follows that functions in $\mathscr{A}_{r}$ map concentric circles onto concentric circles. Such a question was raised by Nitsche [12] for univalent harmonic ring mappings and was negatively answered in [6].

Theorem 2.3 is an existence and uniqueness result for a fixed given dilatation function $a$. This is possible, since the cluster set of a boundary point of $A(r, 1)$ is either a singleton or an interval on the circle $|w|=R$ (resp. $|w|=1)$.

We shall use the following notation. Denote by $\mathscr{P}_{r}$ the class

$$
\begin{aligned}
& \mathscr{P}_{r}=\{p \in H(A(r, 1)) ; \operatorname{Re} p>0 \text { on } A(r, 1) \text { and } \\
&\left.\frac{1}{2 \pi i} \oint_{|z|=\rho} p(z) \frac{d z}{z}=1 \text { and for some } \rho \in(r, 1)\right\} .
\end{aligned}
$$

This class has been studied by several authors, for example, in $[3,7-10,13]$. In particular, mappings in $\mathscr{P}_{r}$ can be represented by the generalized Herglotz formula

$$
p(z)=\int_{-\pi}^{\pi} p^{*}\left(z e^{-i t}\right) d \mu(t)+\int_{-\pi}^{\pi} p^{*}\left(\frac{r}{z e^{-i t}}\right) d \nu(t)-1
$$

where

$$
p^{*}(z)=\frac{1+z}{1-z}+2 \sum_{1}^{\infty} \frac{r^{2 k}}{1-r^{2 k}}\left(z^{k}-z^{-k}\right)
$$

and where $\mu$ and $\nu$ are probability measures on the Borel $\sigma$-algebra over $[-\pi, \pi)$. Note that $(1.3)$ reduces to the classical Herglotz formula if $r=0$. The mapping $p^{*}$ is univalent on $A(r, 1)$ and maps $A(r, 1)$ onto the right halfplane cut along a vertical slit, which lies on $\{w ; \operatorname{Re} w \equiv 1\}$ and is bisected by the real axis. 
Let $S^{*}(r, 1)$ be the set of all univalent analytic functions $\varphi$ on $A(r, 1)$ having the properties:

(i) $p(z)=z \varphi^{\prime}(z) / \varphi(z) \in H(A(r, 1))$,

(ii) $\operatorname{Re} p(z)>0$ on $A(r, 1)$.

The condition of $\varphi$ to be univalent can be replaced by the property $p(z)=$ $1+\sum_{k \in Z \backslash\{0\}} p_{k} z^{k}$. For completeness we give a short proof.

Lemma 1.1. $\varphi \in S^{*}(r, 1)$ if and only if $p(z)=z \varphi^{\prime}(z) / \varphi(z) \in \mathscr{P}_{r}$.

Proof. If $\varphi \in S^{*}(r, 1)$, it is sufficient to show that $p_{0}=1$. Indeed, we have for $\rho \in(r, 1)$

$$
p_{0}=\frac{1}{2 \pi i} \oint_{|z|=\rho} \frac{z \varphi^{\prime}(z)}{\varphi(z)} \frac{d z}{z}=\frac{1}{2 \pi} \oint_{|z|=\rho} d \arg \varphi\left(\rho e^{i t}\right)=1 .
$$

Next, suppose that $p(z)=z \varphi^{\prime}(z) / \varphi(z)=1+\sum_{k \in Z \backslash\{0\}} p_{k} z^{k} \in H(A(r, 1))$ and that $\operatorname{Re} p(z)>0$ on $A(r, 1)$. Then $\varphi \in H(A(r, 1))$. We show that $\varphi$ is univalent on $A(r, 1)$. Fix $\rho \in(r, 1)$. Since $\operatorname{Re} p(z)>0$ on $A(r, 1)$ and $p_{0}=1$, we have

$$
\frac{1}{2 \pi} \oint_{|z|=\rho} d \arg z \varphi^{\prime}(z)=\frac{1}{2 \pi} \oint_{|z|=\rho} d \arg \varphi(z)=p_{0}=1 .
$$

Moreover, we conclude from the relation

$$
\frac{\partial \arg \varphi\left(\rho e^{i t}\right)}{\partial t}=\operatorname{Re}\left\{\frac{\rho e^{i t} \varphi^{\prime}\left(\rho e^{i t}\right)}{\varphi\left(\rho e^{i t}\right)}\right\}>0
$$

that $\varphi$ is univalent on $\{z:|z|=\rho\}$ and that $\Gamma_{\rho}=\left\{w: w=\varphi\left(\rho e^{i t}\right)\right.$; $0 \leq t \leq 2 \pi\}$ is a simple closed strictly starlike curve winding once around the origin. Finally, for $r<\rho_{1}<\rho_{2}<1$, we have $\Gamma_{\rho_{1}} \cap \Gamma_{\rho_{2}}=\varnothing$. Indeed, $\varphi\left(\rho_{1}<|z|<\rho_{2}\right)$ has to be a bounded domain. Therefore, $\varphi$ is univalent on $\left\{z: \rho_{1}<|z|<\rho_{2}\right\}$ for all $r<\rho_{1}<\rho_{2}<1$ and, hence, also on $A(r, 1)$.

Let $f$ be in $\mathscr{A}_{r}$. Then $F(\zeta)=\log f\left(e^{\zeta}\right)$ is a univalent harmonic mapping from the vertical strip $\mathscr{S}_{c}=\{\zeta:-c<\operatorname{Re} \zeta<0\}, c=-\ln r$, onto the vertical strip $S_{-\log R}$. Furthermore, $F$ is a solution of the elliptic partial differential equation

$$
\overline{F_{\bar{\zeta}}}=a F_{\zeta},
$$

where the second dilatation function $a$ satisfies

$$
a \in H\left(\mathscr{S}_{c}\right),|a|<1 \text {, and } a(\zeta+2 \pi i)=a(\zeta) \text { on } \mathscr{S}_{c} \text {. }
$$

Such mappings $F$ appear in a natural way in studying the nonparametric minimal surfaces over a vertical strip whose normal directions are periodic. (For more details see $[4,12]$.)

Suppose now that $a$ satisfies (1.6) and that $F=U+i V$ is a univalent solution of (1.5) which maps $\mathscr{S}_{c}$ onto $\mathscr{S}_{d}$ for some $d>0$ such that $\lim _{\xi \uparrow 0} U(\xi+i \eta) \equiv 0$ and $\lim _{\xi \downarrow-c} U(\xi+i \eta) \equiv-d$. We show in Theorem 3.1 that there is a $\delta>0$ such that the function $f(z)=\exp \left[\frac{2 \pi}{\delta} F(\log z)\right]$ belongs to $\mathscr{A}_{r}$. 
In what follows we shall frequently use the expression $|z|^{2 \beta}$. We always take the branch corresponding to $1^{2 \beta}=1$.

\section{LOGHARMONIC RING MAPPINGS}

We start with a characterization of univalent logharmonic ring mappings.

Theorem 2.1. A function $f$ belongs to $\mathscr{A}_{r}$ if and only if

$$
f(z)=z|z|^{2 \beta} h(z) / \overline{h(z)},
$$

where

(a) $h \in H(A(r, 1))$ and $0 \notin h(A(r, 1))$;

(b) $\operatorname{Re}\left(z h^{\prime}(z) / h(z)\right)>-1 / 2$ on $A(r, 1)$;

(c) $\frac{1}{2 \pi} \int_{|z|=\rho} d \arg h(z)=0, r<\rho<1$;

(d) $\operatorname{Re} \beta>-1 / 2$.

In particular, functions belonging to $\mathscr{A}_{r}$ map concentric circles onto concentric circles.

Proof. Let $f \in \mathscr{A}_{r}$, and suppose that $f(A(r, 1))=A(R, 1)$. Then we have $f(z)=H(z) \overline{G(z)}$ where $H$ and $G$ are locally analytic (possibly multivalued) functions on $A(r, 1)$. Furthermore, because $f(z) \neq 0$ on $A(r, 1)$, the functions

$$
\frac{f_{z}}{f}=\frac{H^{\prime}}{H}=\sum_{k \in Z} a_{k} z^{k}, \quad \frac{\bar{f}_{\bar{z}}}{\bar{f}}=\frac{G^{\prime}}{G}=\sum_{k \in Z} b_{k} z^{k}
$$

are analytic on $A(r, 1)$. By integration and exponentiation we get formally

$$
f(z)=z^{a-1} \bar{z}^{\overline{b-1}} h(z) \overline{g(z)}
$$

where $h$ and $g$ belong to $H(A(r, 1))$ and satisfy

$$
\frac{1}{2 \pi} \oint_{|z|=\rho} d \arg h(z)=\frac{1}{2 \pi} \oint_{|z|=\rho} d \arg g(z)=0
$$

for all $\rho \in(r, 1)$. Therefore, the conditions (a) and (c) hold.

The identity $f\left(z e^{2 \pi i}\right) \equiv f(z)$ implies that $e^{2 \pi i\left(a_{-1}-\overline{b_{-1}}\right)}=1$ and that $a_{-1}$ $\overline{b_{-1}}=n \in Z$. So far, we have the representation

$$
f(z)=z^{n}|z|^{2 \beta} h(z) \overline{g(z)}, \quad n \in Z, \beta=\overline{b_{-1}} \text {. }
$$

Since

$$
|h \bar{g}|_{|z|=r} \equiv R / r^{n+2 \operatorname{Re} \beta} \quad \text { and }|h \bar{g}|_{|z|=1} \equiv 1 \text {, }
$$

we conclude that $h g=e^{i \alpha} z^{m}, \alpha \in \mathscr{R}, m \in Z$. But (2.2) shows that $m=0$, and we are lead to the representation

$$
f(z)=e^{i \alpha} z^{n}|z|^{2 \beta} \frac{h(z)}{\overline{h(z)}}, \quad h \in H(A(r, 1)) .
$$

Finally, we have

$$
1=\oint_{|z|=\rho} d \arg f(z)=n+2 \oint_{|z|=\rho} d \arg h(z)=n
$$


for all $\rho \in(r, 1)$, from which it follows that $n=1$. Replacing $h(z)$ by $e^{-i \alpha / 2} h(z)$, we get the representation formula

$$
f(z)=z|z|^{2 \beta} h(z) / \overline{h(z)} .
$$

The relation (2.1) implies that $\left|f\left(r e^{i \theta}\right)\right|=R=r^{1+2 \operatorname{Re} \beta}$, and the relation $\operatorname{Re} \beta=(1 / 2)[(\log R) / \log r-1]>-1 / 2$ shows that (d) holds. In order to prove (b), first observe that $|f(z)|_{|z|=\rho}=\rho^{1+2 \operatorname{Re} \beta}, \rho \in(r, 1)$. Since $f$ is univalent on $A(r, 1)$ and $\oint_{|z|=\rho} d \arg f(z)=1$, we get for $z=\rho e^{i t} \in A(r, 1)$

$$
\begin{aligned}
\frac{\partial \arg f\left(\rho e^{i t}\right)}{\partial t} & =\operatorname{Im}\left(i \frac{z f_{z}}{f}-i \frac{\bar{z} f_{\bar{z}}}{f}\right)=\operatorname{Re}\left(\frac{z f_{z}}{f}-\frac{\bar{z} f_{\bar{z}}}{f}\right) \\
& =\operatorname{Re}\left(z\left(\frac{1+\beta}{z}+\frac{h^{\prime}}{h}-\frac{\beta}{z}+\frac{h^{\prime}}{h}\right)\right)=\operatorname{Re}\left(1+2 \frac{z h^{\prime}(z)}{h(z)}\right)>0,
\end{aligned}
$$

and $(b)$ is proved. The necessity part is established.

Conversely, assume that $f(z)=z|z|^{2 \beta} h(z) / \overline{h(z)}$ and that the conditions (a)(d) are satisfied. We have to prove that $f$ is a univalent logharmonic mapping from $A(r, 1)$ onto $A(R, 1)$ for some $R \in(0,1)$ and that $\oint_{|z|=\rho} d \arg f(z)=1$ for all $\rho \in(r, 1)$. First of all, we see that $f$ maps any circle $\{|z|=\rho\}$, $\rho \in(r, 1)$, into the circle $\left\{|w|=\rho^{1+2 \operatorname{Re} \beta}\right\}$. Let us show that $f$ is a solution of (1.1). By (2.1), we have

$$
a=\frac{\overline{f_{\bar{z}}} / \bar{f}}{f_{z} / f}=\frac{\bar{\beta}-z h^{\prime} / h}{1+\beta+z h^{\prime} / h} \in H(A(r, 1)),
$$

and, since $\operatorname{Re} \beta>-1 / 2$ and $\operatorname{Re}\left(z h^{\prime}(z) / h(z)\right)>-1 / 2$ on $A(r, 1)$, elementary calculations show that $|a|<1$ on $A(r, 1)$. Next, we show that $f$ is univalent on $A(r, 1)$. Using the relation $(2.5)$ and condition (b), we conclude that each circle $\{z:|z|=\rho\}, \rho \in(r, 1)$, is mapped monotonically onto the circle $\left\{w:|w|=\rho^{1+2 \operatorname{Re} \beta}\right\}$, and, from condition (c), we deduce that $f$ is univalent in $A(r, 1)$ and that $\oint_{|z|=\rho} d \arg f=1$. Therefore, $f \in \mathscr{A}_{r}$. This completes the proof.

Another version of Theorem 2.1 is the following representation.

Theorem 2.2. A function $f$ is in $\mathscr{A}_{r}$ if and only if it is of the form

$$
f(z)=\left(\frac{\varphi(z)}{|\varphi(z)|}|z|^{2 \gamma}\right),
$$

where $\operatorname{Re} \gamma>0$ and $\varphi \in S^{*}(r, 1)$.

Proof. Let $f$ be in $\mathscr{A}_{r}$. Then, by Theorem 2.1, we have

$$
f(z)=z|z|^{2 \beta} h(z) / \overline{h(z)},
$$

where $\operatorname{Re} \beta>-1 / 2, \operatorname{Re}\left(z h^{\prime}(z) / h(z)\right)>-1 / 2$ on $A(r, 1)$, and $\int_{|z|=\rho} d \arg h(z)$ $=0$ for all $\rho \in(r, 1)$. Put $\varphi(z)=z h^{2}(z)$. It follows that

$$
f(z)=z|z|^{2 \beta} \frac{h^{2}(z)}{|h(z)|^{2}}=\left(\frac{\varphi(z)}{|\varphi(z)|}|z|^{2 \beta+1}\right)=\left(\frac{\varphi(z)}{|\varphi(z)|}|z|^{2 \gamma}\right),
$$


where $\operatorname{Re} \gamma>0$. On the other hand, we have

$$
\operatorname{Re}\left(z \varphi^{\prime}(z) / \varphi(z)\right)=1+2 \operatorname{Re}\left(z h^{\prime}(z) / h(z)\right)>0
$$

for all $z \in A(r, 1)$. Moreover, putting $p(z)=z \varphi^{\prime}(z) / \varphi(z)$ we get

$$
\begin{aligned}
p_{0} & =\frac{1}{2 \pi i} \oint_{|z|=\rho} \frac{p(z)}{z} d z=\frac{1}{2 \pi i} \oint_{|z|=\rho} \frac{\varphi^{\prime}(z)}{\varphi(z)} d z=\frac{1}{2 \pi} \oint_{|z|=\rho} d \arg \varphi(z) \\
& =1+\frac{1}{\pi} \oint_{|z|=\rho} d \arg h(z)=1,
\end{aligned}
$$

which implies (Lemma 1.1) that $\varphi \in S^{*}(r, 1)$.

Conversely, let $f=|z|^{2 \gamma} \varphi /|\varphi|, \operatorname{Re} \gamma>0$, and $\varphi \in S^{*}(r, 1)$. We have to show that $h(z)=\sqrt{\varphi(z) / z}$ can be defined as a single-valued function on $A(r, 1)$. Observe first that

$$
\frac{z h^{\prime}}{h}=\frac{\left[z \varphi^{\prime} / \varphi-1\right]}{2} \in H(A(r, 1)) .
$$

By (2.9), we have

$$
\frac{1}{2 \pi} \oint_{|z|=\rho} d \arg h=\frac{1}{2}\left\{\left[\frac{1}{2 \pi} \oint_{|z|=\rho} d \arg \varphi\right]-1\right\}=\frac{p_{0}-1}{2}=0 .
$$

Therefore, we can define $\log h$ and, hence, also $h$ as an analytic function on $A(r, 1)$. Evidently, $h$ does not vanish on $A(r, 1)$; so we have shown properties (a) and (c) of $h$ in Theorem 2.1. The properties (b) and (d) of $h$ and the form (2.1) of $f$ follow from the relations (2.7) and (2.8). Finally, we apply Theorem 2.1 and the proof is complete.

For the next result, we fix the second dilatation function $a \in H(A(r, 1))$, $|a(z)|<1$ for all $z \in A(r, 1)$.

Theorem 2.3. For a given $a \in H(A(r, 1)),|a(z)|<1$ for all $z \in A(r, 1)$ and for a given $z_{0} \in A(r, 1)$, there exists one and only one univalent solution $f$ of (1.1) in $\mathscr{A}_{r}$ such that $f\left(z_{0}\right)>0$.

Remark. Theorem 2.3 is not true for univalent harmonic ring mappings (see [6, Theorem 7.3]).

In the proof of Theorem 2.3 we shall need the following lemma.

Lemma 2.4. There exists a $\beta$ depending on a such that $\operatorname{Re} \beta>-1 / 2$ and

$$
I=\int_{0}^{2 \pi} \frac{\bar{\beta}-(1+\beta) a\left(\rho e^{i t}\right)}{1+a\left(\rho e^{i t}\right)} d t=0
$$

for all $\rho \in(r, 1)$.

Proof. Observe that $I$ does not depend on $\rho$ since the integrand is analytic on $A(r, 1)$. Furthermore (writing $\left.a\left(\rho e^{i t}\right)=a\right)$, the condition

$$
I=\int_{0}^{2 \pi} \frac{\bar{\beta}-|a|^{2}-\beta a-a+\overline{a \beta}-\beta|a|^{2}}{|1+a|^{2}} d t=0
$$

is satisfied for

$$
\operatorname{Re} \beta=\left(\int_{0}^{2 \pi} \frac{\operatorname{Re} a+|a|^{2}}{|1+a|^{2}} d t\right)\left(\int_{0}^{2 \pi} \frac{1-|a|^{2}}{|1+a|^{2}} d t\right)^{-1}
$$


and

$$
\operatorname{Im} \beta=-\frac{(1+2 \operatorname{Re} \beta)}{2 \pi} \int_{0}^{2 \pi} \frac{\operatorname{Im} a}{|1+a|^{2}} d t .
$$

Furthermore, we have

$$
1+2 \operatorname{Re} \beta=2 \pi\left(\int_{0}^{2 \pi} \frac{1-|a|^{2}}{|1+a|^{2}} d t\right)^{-1}>0,
$$

and the lemma is shown.

Proof of Theorem 2.3. Consider the function

$$
h(z)=\exp \left(\int \frac{\bar{\beta}-(1+\beta) a(z)}{z(1+a(z))} d z\right),
$$

where $\beta$ is defined in Lemma 2.4. Since

$$
\frac{1}{2 \pi} \oint_{|z|=\rho} d \arg h(z)=\frac{1}{2 \pi i} \oint_{|z|=\rho} \frac{\bar{\beta}-(1+\beta) a(z)}{z(1+a(z))} d z=\frac{I}{2 \pi}=0,
$$

it follows that $h \in H(A(r, 1))$ and that

$$
\operatorname{Re}\left(\frac{z h^{\prime}(z)}{h(z)}+\frac{1}{2}\right)=\frac{1-|a|^{2}}{|1+a|^{2}}\left(\frac{1}{2}+\operatorname{Re} \beta\right)>0 \text { on } A(r, 1) .
$$

This implies that $\operatorname{Re}\left(z h^{\prime}(z) / h(z)\right)>-1 / 2$ on $A(r, 1)$. Applying Theorem 2.1, we conclude that the function $\hat{f}$ defined by

$$
\hat{f}(z)=z|z|^{2 \beta} h(z) / \overline{h(z)}
$$

belongs to $\mathscr{A}_{r}$ and is a solution of (1.1). Finally, take

$$
f(z)=\left(\overline{\hat{f}\left(z_{0}\right)} /\left|\hat{f}\left(z_{0}\right)\right|\right) \hat{f}(z),
$$

and the proof of the existence is established.

We now show the uniqueness of the mapping. Suppose that $f_{1}$ and $f_{2}$ are two solutions of (1.1) satisfying the conditions of Theorem 2.3.

First, we show that $f_{1}\left(z_{0}\right)=f_{2}\left(z_{0}\right)$, which implies that $\operatorname{Re} \beta$ is unique, since $f_{1}\left(z_{0}\right)=f_{2}\left(z_{0}\right)=r^{1+2 \operatorname{Re} \beta}$.

Suppose that the contrary holds. Put

$$
\frac{f_{1}\left(z_{0}\right)}{f_{2}\left(z_{0}\right)}=\left|z_{0}\right|^{2 \operatorname{Re}\left(\beta_{1}-\beta_{2}\right)}=s>0 \text { and } \Phi(z)=s \cdot \frac{f_{2}(z)}{f_{1}(z)} .
$$

The function $\Phi$ is again a nonconstant solution of (1.1) with respect to the same dilatation function $a$ and is hence an orientation-preserving open mapping. We have $\left|\Phi\left(\rho e^{i t}\right)\right|=\left(\rho /\left|z_{0}\right|\right)^{2 \operatorname{Re}\left(\beta_{2}-\beta_{1}\right)}$ for all $\rho \in(r, 1)$. With no loss of generality, we may assume that $\operatorname{Re}\left(\beta_{2}-\beta_{1}\right)>0$; if not, consider $1 / \Phi$. Then we have $\left|\Phi\left(e^{i t}\right)\right|>1$ and $\left|\Phi\left(r e^{i t}\right)\right|<1$. Choose $r<\rho_{1}<\rho_{2}<1$ such that $\left|\Phi\left(\rho_{1} e^{i t}\right)\right|<1$ and $\left|\Phi\left(\rho_{2} e^{i t}\right)\right|>1$. Then, by the argument principle, we have

$$
J=\frac{1}{2 \pi} \oint_{|z|=\rho_{2}} d \arg (\Phi(z)-1)-\frac{1}{2 \pi} \oint_{|z|=\rho_{1}} d \arg (\Phi(z)-1) \geq 1,
$$


since $\rho_{1}<\left|z_{0}\right|<\rho_{2}$ and $\Phi\left(z_{0}\right)=1$. On the other hand,

$$
\frac{1}{2 \pi} \oint_{|z|=\rho_{2}} d \arg \left(f_{1}\right)-\frac{1}{2 \pi} \oint_{|z|=\rho_{1}} d \arg \left(f_{1}\right)=0
$$

and, therefore,

$$
J=\frac{1}{2 \pi} \oint_{|z|=\rho_{2}} d \arg \left(s f_{2}-f_{1}\right)-\frac{1}{2 \pi} \oint_{|z|=\rho_{1}} d \arg \left(s f_{2}-f_{1}\right) .
$$

Since, for $\left|z_{0}\right| \neq \rho$,

$$
s\left|f_{2}\left(\rho e^{i t}\right)\right|-\left|f_{1}\left(\rho e^{i t}\right)\right|=\rho^{1+2 \operatorname{Re}\left(\beta_{1}\right)}\left(\left(\frac{\left|z_{0}\right|}{\rho}\right)^{2 \operatorname{Re}\left(\beta_{1}-\beta_{2}\right)}-1\right) \neq 0,
$$

we conclude that $J=0$, which leads to a contradiction. Hence, $f_{1}\left(z_{0}\right)=$ $f_{2}\left(z_{0}\right)$, which implies that $|\phi| \equiv 1$ on $A(r, 1)$ and $\Phi\left(z_{0}\right)=1$. Since $\Phi$ is a solution of (1.1) but is not an open mapping, it follows that $\Phi \equiv 1$, and, therefore, $f_{1} \equiv f_{2}$.

The next result gives an algebraic structure of the class $\mathscr{A}_{r}$.

Theorem 2.5. The family $\mathscr{A}_{r}$ is "logarithmically convex", i.e., if $f_{1}, f_{2} \in \mathscr{A}_{r}$, then $F=f_{1}^{\lambda} f_{2}^{1-\lambda} \in \mathscr{A}_{r}$ for all $\lambda \in(0,1)$.

Proof. Let $\varphi_{j}, j=1,2$, be the univalent starlike mappings defined in Theorem 2.2 corresponding to $f_{j}, j=1,2$. Then the functions $p_{j}(z)=$ $z \varphi_{j}^{\prime}(z) / \varphi_{j}(z)$ belong to $\mathscr{P}_{r}$. Since $p=\lambda p_{1}+(1-\lambda) p_{2}$ has the same property, we conclude from Lemma 1.1 that $\varphi=\varphi_{1}^{\lambda} \varphi_{2}^{1-\lambda} \in S^{*}(r, 1)$. Finally, by Theorem 2.2, we have

$$
f(z)=f_{1}^{\lambda}(z) \cdot f_{2}^{1-\lambda}(z)=\left(\frac{\varphi(z)}{|\varphi(z)|}|z|^{2\left(\lambda \gamma_{1}+(1-\lambda) \gamma_{2}\right)}\right)=\left(\frac{\varphi(z)}{|\varphi(z)|}|z|^{2 \gamma}\right)
$$

for all $z \in A(r, 1)$, and, therefore, $f$ belongs to $\mathscr{A}_{r}$.

\section{UNIVALENT HARMONIC STRIP MAPPINGS WITH PERIODIC DILATATIONS}

Denote by $\mathscr{S}_{c}$ the vertical strip $\{\zeta ;-c<\operatorname{Re} \zeta<0\}, \zeta=\xi+i \eta$, and let $\Sigma_{c}$ be the set of all univalent harmonic and orientation-preserving mappings $F=U+i V$ from $\mathscr{S}_{c}$ onto $\mathscr{S}_{d}$ for some $d>0$ such that $\lim _{\xi \uparrow 0} U(\xi+i \eta) \equiv 0$ and $\lim _{\xi 1-c} U(\xi+i \eta) \equiv-d$. It follows that $U(\zeta)=d \xi / c$ and that there is an $a \in H\left(\mathscr{S}_{c}\right),|a|<1$ on $\mathscr{S}_{c}$, such that $F$ is a solution of the elliptic partial differential equation

$$
\overline{F_{\bar{\zeta}}}=a F_{\zeta} .
$$

Furthermore, $F$ admits the representation

$$
F(\zeta)=\frac{d}{c}\left[\xi+i \operatorname{Im} \int^{\zeta} \frac{1-a(s)}{1+a(s)} d s\right] .
$$

Indeed, (3.1) is equivalent to the equation

$$
i V_{\zeta}=\frac{1-a}{1+a} U_{\zeta} .
$$


Let $\mathscr{F}_{c}$ be the set of all mappings $F$ which are of the form (3.2). First, we observe that each $F \in \mathscr{F}_{c}$ is univalent harmonic and orientation-preserving on $\mathscr{S}_{c}$. Evidently, $F$ is harmonic, and we conclude from

$$
\overline{F_{\bar{\zeta}}}=\frac{d}{c} \cdot \frac{a}{1+a} \text { and } F_{\zeta}=\frac{d}{c} \cdot \frac{1}{1+a}
$$

that $F$ is a solution of $(3.1)$ and, therefore, is orientation-preserving. The univalence of $F$ follows from the fact that the vertical lines $\left\{\zeta: \operatorname{Re} \zeta=\xi_{0}\right\}$, $\xi_{0} \in(-c, 0)$, are mapped into the vertical lines $\left\{w: \operatorname{Re} w=d \xi_{0} / c\right\}$ and that $\frac{\partial V}{\partial \eta}=\operatorname{Re} \frac{(1-a)}{1+a}>0$ on $\mathscr{S}_{c}$.

There are mappings in $\mathscr{F}_{c}$ which do not belong to $\Sigma_{c}$. For instance,

$$
F(\zeta)=\xi-\frac{i}{2} e^{2 \eta} \sin (2 \xi)
$$

belongs to $\mathscr{F}_{\pi / 2}$ but not to $\Sigma_{\pi / 2}$. Indeed, $F$ is of the form (3.2) with

$$
a(s)=\frac{1+i e^{-2 i s}}{1-i e^{-2 i s}}, \quad s \in \mathscr{S}_{\pi / 2}
$$

and

$$
F\left(\mathscr{S}_{\pi / 2}\right)=\{w ;-\pi / 2<\operatorname{Re} w<0 \text { and } \operatorname{Im} w>0\}
$$

is not a vertical strip. On the other hand, by similar arguments as in [5, Theorem 2.7], $\Sigma_{c}$ is dense in $\mathscr{F}_{c}$ with respect to the topology of the locally uniform convergence.

Suppose now that $a$ satisfies the periodicity condition

$$
a(\zeta+2 \pi i)=a(\zeta), \quad \zeta \text { in } \mathscr{S}_{c}
$$

Then (3.2) defines a univalent solution of (3.1), which maps $\mathscr{S}_{c}$ into $\mathscr{S}_{d}$. Let us show that $F=U+i V \in \Sigma_{c}$, i.e., that $F$ maps $\mathscr{S}_{c}$ onto $\mathscr{S}_{d}$. Indeed, $F(\zeta+2 \pi i)$ is also a univalent solution of (3.1), and, since

$$
F(\zeta+2 \pi i)-F(\zeta)=\frac{d}{c} i \operatorname{Im} \int_{\zeta}^{\zeta+2 \pi i} \frac{1-a(s)}{1+a(s)} d s
$$

is not an open mapping, it follows that

$$
F(\zeta+2 \pi i) \equiv F(\zeta)+i \delta \quad \text { on } \mathscr{S}_{c}
$$

for some $\delta \in \mathscr{R}$. The univalence of $F$ and the property $\partial V / \partial \eta>0$ imply that $\delta>0$, from which we conclude that the vertical lines $\left\{\zeta: \operatorname{Re} \zeta=\xi_{0}\right\}$, $\xi_{0} \in(-c, 0)$, are mapped onto the vertical lines $\left\{w: \operatorname{Re} w=d \xi_{0} / c\right\}$.

Summarizing we have shown:

Theorem 3.1. Let $a \in H\left(\mathscr{S}_{c}\right),|a|<1$, and $a(\zeta+2 \pi i)=a(\zeta)$ on $\mathscr{S}_{c}$. Then (3.2) defines a univalent solution $F$ of (3.1) which belongs to $\Sigma_{c}$. Furthermore, any solution of (3.1) which belongs to $\Sigma_{c}$ is of the form (3.2), and there is a $\delta>0$ such that (3.3) holds.

Define

$$
\Sigma_{c, \mathscr{P}}=\left\{F \in \Sigma_{c} ; a(\zeta+2 \pi i)=a(\zeta) \text { on } \mathscr{S}_{c}\right\}
$$


For $F \in \Sigma_{c, \mathscr{P}}$, consider the mapping

$$
f(z)=\exp \left[\frac{2 \pi}{\delta} F(\log z)\right],
$$

where $\delta$ is defined in (3.3). Then $f$ is a univalent and logharmonic mapping on $A\left(e^{-c}, 1\right)$ and belongs to the class $\mathscr{A}_{r}, r=e^{-c}$. Conversely, if $f \in \mathscr{A}_{r}$ then $F(\zeta)=\log f\left(e^{\zeta}\right) \in \Sigma_{c, \mathscr{D}}, c=-\log r$. It is easy to transfer results from one class to the other. For instance, we may get a second proof of the existence in Theorem 2.3 as follows:

Let $a_{1} \in H(A(r, 1)),\left|a_{1}\right|<1$ on $A(r, 1)$, and let $z_{0} \in A(r, 1)$ be given. Put $a(s)=a_{1}\left(e^{s}\right)$, and let $F$ be defined by (3.2) and $f$ by (3.4). Then $\left(\overline{f\left(z_{0}\right)} /\left|f\left(z_{0}\right)\right|\right) \cdot f$ is the desired solution.

Observe that $\Sigma_{c, \mathscr{P}}$ and $\mathscr{A}_{r}$ are not compact families with respect to the topology of locally uniform convergence. However, we may still estimate some functionals over the classes $\Sigma_{c, \mathscr{P}}$ and $\mathscr{A}_{r}$, since $\mathscr{P}_{r}$ is compact. For instance, the problem

$$
M(\rho, r)=\max _{f \in \mathscr{S}_{*}(r, 1)}\left\{\max _{0 \leq t \leq 2 \pi}\left\{\left|\arg e^{-i t} f\left(\rho e^{i t}\right)\right| \bmod 2 \pi\right\}\right\}, \quad r<\rho<1,
$$

has been solved in [3]. Using Theorem 2.2, we get immediately that

$$
\left|\arg e^{-i t} f\left(\rho e^{i t}\right)\right|_{\bmod 2 \pi} \leq M(r, \rho)+2|\operatorname{Im} \gamma| \ln \rho
$$

for all $f \in \mathscr{A}_{r}$.

\section{REFERENCES}

1. Z. Abdulhadi and D. Bshouty, Univalent mappings in $H \cdot \bar{H}(D)$, Trans. Amer. Math. Soc. 305 (1988), 841-849.

2. Z. Abdulhadi and W. Hengartner, Spiral-like logharmonic mappings, Complex Variables Theory Appl. 9 (1987), 121-130.

3. L. E. Dundučenko, Certain extremal properties of analytic functions given in a circle and in a circular ring, Ukrain. Mat. Zh. 8 (1956), 377-395.

4. W. Hengartner and G. Schober, Curvature estimates for some minimal surfaces, Complex Analysis, Birkhäuser-Verlag, Basel, 1988, pp. 87-100.

5. —_ Univalent harmonic functions, Trans. Amer. Math. Soc. 299 (1987), 1-31.

6. $\ldots$, Univalent harmonic exterior and ring mappings, J. Math. Anal. Appl. 156 (1991), 154-171.

7. Y. Komatu, On analytic functions with positive real part in an annulus, Kodai Math. Rep. 10 (1958), 84-100.

8. Rep. 10 (1958), 145-160.

9. A. E. Livingston and J. A. Pfaltzgraff, Structure and extremal problems for classes of functions in an annulus, Colloq. Math. 43 (1980), 161-181.

10. H. Nishimiya, On coefficients regions of Laurent series with positive real part, Kodai Math. Rep. 11 (1959), 25-39.

11. J. C. C. Nitsche, On the module of doubly connected regions under harmonic mappings, Amer. Math. Monthly 69 (1962), 781-782. 
12. __ Vorlesungen über Minimalflächen, Springer-Verlag, Berlin, 1975.

13. V. A. Zmorovič, On some classes of analytic functions univalent in a circular ring, Mat. Sb. 32 (1953), 633-652.

Department of Mathematical Sciences, King Fahd University of Petroleum and Minerals, Dhahran, Saudi Arabia

E-mail address, Z. Abdulhad: fac1004@saupm00.bitnet

Département de Mathématiques et Statistiques, Université laval, Cité UniversiTAIRE, QuÉbec, P.Q., CANADA G1K 7P4

E-mail address, W. Hengartner: walheng@mat.ulaval.ca

Département de MathématiQues, Faculté des Sciences, Université Monastir, Tunisia Poland

Current address, J. Szynal: Department of Mathematics, University Marie Curie, Lublin, 